\title{
Effect of morphological preservation artifacts on the quality of immuno- histochemical staining for ER and Her-2
}

\author{
Peiris HH ${ }^{1}$, Mudduwa LKB ${ }^{2}$, Liyanage $\mathrm{T}^{2}$, Ranasinghe $\mathrm{JGS}^{3}$ \\ ${ }^{\prime}$ Allied Health Sciences Degree Programme, ${ }^{2}$ Department of Pathology, Faculty of Medicine, University of \\ Ruhuna, Galle, Sri Lanka \\ ${ }^{3}$ Department of Biochemistry, University of Peradeniya, Peradeniya, Sri Lanka
}

Correspondence: Ms. H. Harshini Peiris;

Mobile:+94718332615; e-mail:bbharshi@yahoo.com

\begin{abstract}
Introduction: The most commonly encountered morphological preservation artifacts in the histological assessment of breast cancer include retraction of tumour cell clusters from the stroma, cytoplasmic retraction and alterations in nuclear morphology. This study intended to assess how these artifacts affect the quality of immunohistochemical (IHC) staining for ER and Her-2.

Methods: For this retrospective study, 120 cases of consecutive breast cancers reported by the $2^{\text {nd }}$ and $3^{\text {rd }}$ authors during 2009 to 2010 were included. Haemotoxylin and Eosin stained sections of each breast cancer were assessed to obtain a consensus score for the degree of morphological preservation artifacts present. For each artifact (Retraction of tumour cell clusters, cytoplasmic retraction and alterations in nuclear morphology) a consensus score was given $(0,1,2,3)$ depending on the percentage of cell clusters/cells affected. Each IHC slide was assessed to obtain a consensus score for the quality of IHC staining for ER and Her-2. Parameters measured were uniformity of staining $(0,1)$, background staining $(0,1,2)$, and adequacy of counterstaining $(0,1)$.
\end{abstract}

Results: The study included a total of 120 consecutive breast cancer cases. There was no statistically significant relationship between the consensus score for the commonly encountered morphological preservation artifacts and the quality of IHC staining of ER $(p=0.44)$ and Her-2 $(p=0.51)$.

Conclusions: Commonly encountered morphological preservation artifacts have no significant effect on the quality of IHC staining for ER and Her-2 measured in terms of uniformity of staining, background staining and adequacy of counterstaining.

Keywords: Morphological preservation artifacts, Immunohistochemistry

\section{Introduction}

Immunohistochemistry (IHC) is a widely used ancillary test in the histopathological diagnosis and prognostication of tumours (1). The primary advantage of IHC is that antigen can be detected in the context of architectural and cellular morphology of the tissue in contrast to biochemical assay (2). Therefore morphological preservation of tissue at microscopic level is important in the assessment.

Of the most commonly used predictive and prognostic factors of breast cancer, expression of oestrogen receptors (ER), progesterone receptors (PR) and amplification of human epidermal growth factor receptor 2 (Her-2) are assessed by IHC. Adjuvant treatment for breast cancer is based on these predictive and prognostic factors. Hence accurate IHC assessment of ER, PR and Her-2 are extremely important in making the most appropriate therapeutic decision for breast cancer patients. Patients with ER and PR positive tumours are treated with adjuvant hormone therapy while metastatic breast carcinomas expressing strong positivity for 
Her-2 are given trastuzumab as targeted therapy. Hormone receptors are expressed in the nucleus while Her-2 antigen is found in the cell membrane. Hence the morphological preservation of these sites of antigen expression is important for IHC assessment of breast cancers.

Formalin is the most commonly used fixative in IHC (3). The speed of penetration of tissues by $10 \%$ buffered formalin is about $1 \mathrm{~mm} /$ hour (4). Due to the slow penetration of formalin, breast cancer situated deep in a mastectomy specimen does not get fixed adequately, unless it is sliced soon after the surgery, facilitating early contact with the fixative. Poor fixation is the most common cause of morphological preservation artifacts which are detected in $\mathrm{H} \& \mathrm{E}$ stained slides. This study intended to assess how the commonly encountered morphological preservation artifacts affect the quality of IHC staining for ER and Her-2 as markers which are localized in different parts of breast cancer cells.

\section{Methods}

This was a retrospective study. All breast cancers reported by the $2^{\text {nd }}$ and $3^{\text {rd }}$ authors during 2009 to 2010 were retrieved from the files of the Diagnostic Immunohistochemistry Laboratory of our institution.

Manual IHC staining had been performed on the histological sections prepared from $10 \%$ formalin fixed breast cancer tissue embedded in paraffin wax. Streptavidin Biotin method had been used. Dako (Glostrup, Denmark) monoclonal mouse antihuman ER $\alpha$, clone 1D5 (M7047), polyclonal rabbit antihuman c-erbB-2 oncoprotein (A0485) and Universal LSAB2 kit/HRP Rabbit/mouse with Streptavidin/HRP (K0675, K0673) were used for the IHC staining. For each IHC staining a positive control had been used.

Based on the observations made in day to day breast cancer reporting three commonly encountered morphological preservation artifacts were selected for the assessment (Figure 1).
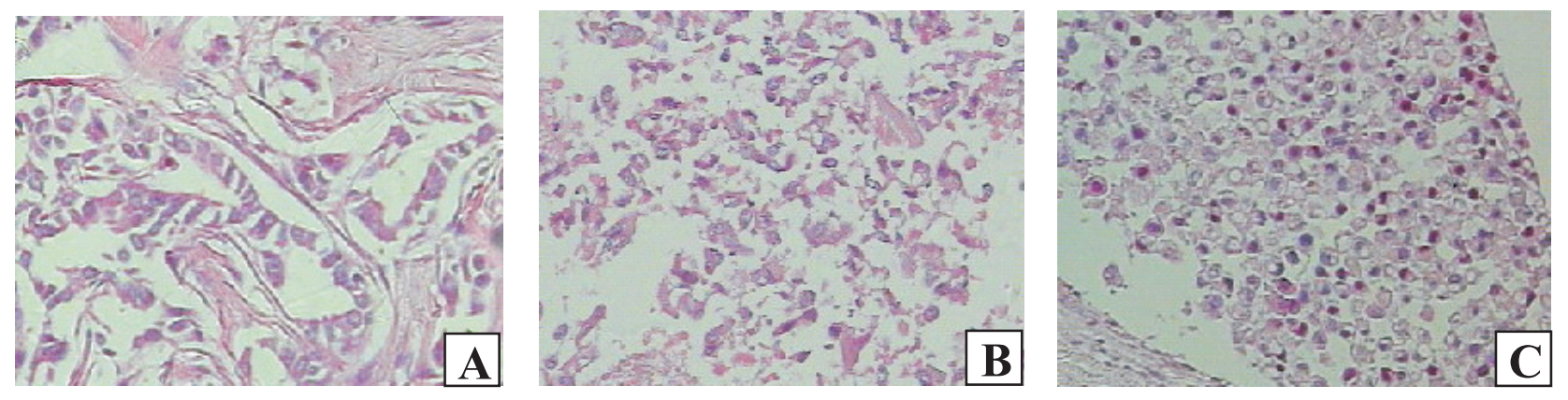

Figure 1: Commonly encountered morphological preservation artifacts in the IHC stained breast cancer tissues. A) retraction of tumour cell clusters from the stroma; B) cytoplasmic retraction; C) alterations in nuclear morphology - shrunken and condensed nuclei.(400x)

These included retraction of tumour cell clusters from the stroma, cytoplasmic retraction and alterations in nuclear morphology. To objectively quantify the artifacts in breast cancer tissue, the investigators devised a scoring system based on the percentage of cells/cluster of cells affected. These three artifacts were assessed separately. When there was no artifact score $=0,<10 \%$ of cells with artifact score $=1,10-75 \%$ cells with artifact score $=2$ and $>75 \%$ of cells with artifact score $=3$.
A Haematoxylin and Eosin stained (H \& E) section of each breast cancer was assessed over a binocular multi-head microscope to obtain a consensus score for the preservation artifacts. Shrunken and condensed nuclei in breast cancer cells were considered the features of alteration in nuclear morphology. In assessing altered nuclear morphology, apoptotic nuclei among preserved breast cancer cells were excluded.

Each IHC slide was assessed for the quality of IHC staining out of a possible score of 4 (Table 1). 
Table 1: Scoring system for quality of IHC staining

\begin{tabular}{llll}
\hline Criteria for scoring & $\mathbf{0}$ & $\mathbf{1}$ & $\mathbf{2}$ \\
\hline $\begin{array}{l}\text { Uniformity of IHC } \\
\text { staining }\end{array}$ & $\begin{array}{l}\text { Not uniform } \\
\text { throughout }\end{array}$ & Uniform throughout & NA \\
Background staining & $\begin{array}{l}\text { Excessive background } \\
\text { staining interfering } \\
\text { with the interpretation } \\
\text { Inadequate }\end{array}$ & $\begin{array}{l}\text { Background staining } \\
\text { with no interference to } \\
\text { the interpretation } \\
\text { Adequate }\end{array}$ & $\begin{array}{l}\text { No background } \\
\text { staining }\end{array}$ \\
$\begin{array}{l}\text { Adequacy of counter } \\
\text { staining }\end{array}$ & NA \\
\hline NA- not applicable. [A modified version of the scoring system developed by Maxwell and McCluggage, 2000]
\end{tabular}

This is a modified version of the scoring system developed by Maxwell and McCluggage in 2000 (5). The intensity of staining and the specificity of staining which were considered in original scoring system were omitted as those two parameters have no value in the assessment of ER negative and Her-2 negative tumours.

Data analysis was done using SPSS Version 11 package. Cases were categorized into three groups (Grade 1, 2 and 3) depending on the total score for commonly encountered preservation artifacts. Analysis of variance was used to compare the mean values of the quality of IHC staining (for ER and Her2 separately) for the above groups. Both scoring systems were pre-tested before using on the study sample.

Ethical approval was obtained from the Ethical Review Committee of our institution, before commencing the study.

\section{Results}

This study included a total of 125 consecutive breast cancer cases. H\&E slides and IHC slides for ER were available for all 125 cases. However, only 123 cases had the corresponding Her-2 stained slides.

There were 103 (82.4\%) mastectomies, 7 (5.6\%) lumpectomies, $1(0.8 \%)$ wide local excision, $9(7.2 \%)$ axillary lymph nodes with metastasis and 5 (4\%) Tru-cut biopsies. Since Tru-cut biopsies are immediately immersed in formalin and do not show preservation artifacts they were excluded from the sample. Therefore a total of 120 breast cancer cases were included in the study.

\section{Commonly encountered morphological preservation artifacts}

The expected total score for the morphological preservation artifacts ranged from 0 to 9 where 0 signified the absence of the assessed artifacts. There were eight cases which had the maximum total score of 9 for the preservation artifacts but none had score 0 (Table 2).

Table 2: Frequency distribution of commonly encountered morphological preservation artifacts total score

\begin{tabular}{lllllllllll}
\hline Score & $\mathbf{1}$ & $\mathbf{2}$ & $\mathbf{3}$ & $\mathbf{4}$ & $\mathbf{5}$ & $\mathbf{6}$ & $\mathbf{7}$ & $\mathbf{8}$ & $\mathbf{9}$ & Total \\
\hline $\begin{array}{l}\text { Frequency } \\
(\%)\end{array}$ & 12 & 18 & 13 & 14 & 11 & 15 & 18 & 11 & 8 & 120 \\
& $(10.0)$ & $(15.0)$ & $(10.8)$ & $(11.7)$ & $(9.2)$ & $(12.5)$ & $(15.0)$ & $(9.2)$ & $(6.7)$ & $(100)$ \\
\hline
\end{tabular}

Depending on the total score for the artifacts, cases were categorized into three groups. A score ranging from 0 - 3 $($ Grade 1$)=$ minor degree of artifacts, $4-6($ Grade 2$)=$ moderate degree of artifacts and $7-9($ Grade 3$)=$ high degree of artifacts (Table 3). 


\section{Quality of IHC staining ER}

IHC stained slides for ER was available for all 120 cases. The best staining quality was indicated by a score of 4 . The majority (63.3\%) of the IHC stained slides scored 3 or 4 and thus indicating good quality of IHC staining (Table 4).

The majority of the cases had uniform staining throughout the section and background staining did not interfere with the interpretation. Most of the cases had adequate counter staining (Table 5).
Table 3: Mean values of the quality of IHC staining for the degree of preservation artifacts

\begin{tabular}{lll}
\hline & $\begin{array}{l}\text { Degree of } \\
\text { preservation } \\
\text { artifacts (grade) }\end{array}$ & Mean (SD) \\
\hline Total Score & $1(\mathrm{n}=43)$ & $2.6(0.8)$ \\
for ER & $2(\mathrm{n}=40)$ & $2.3(1.1)$ \\
& $3(\mathrm{n}=37)$ & $2.5(0.8)$ \\
\hline Total Score & $1(\mathrm{n}=41)$ & $2.7(0.8)$ \\
for Her-2 & $2(\mathrm{n}=40)$ & $2.5(0.9)$ \\
& $3(\mathrm{n}=37)$ & $2.6(0.7)$ \\
\hline
\end{tabular}

Table 4: Score for the quality of IHC staining for ER and Her-2

\begin{tabular}{lll}
\hline Total Score & ER & Her-2 \\
& Frequency (\%) & Frequency (\%) \\
\hline 0 (Poor quality) & $3(2.4)$ & $2(1.6)$ \\
1 & $20(16.0)$ & $13(10.6)$ \\
2 & $24(19.2)$ & $26(21.1)$ \\
3 & $72(57.6)$ & $75(61.0)$ \\
4 (Best quality) & $6(4.8)$ & $7(5.7)$ \\
\hline Total & $\mathbf{1 2 5}$ & $\mathbf{1 2 3}$ \\
\hline
\end{tabular}

Table 5: Quality of IHC staining for ER and Her2; according to the features assessed for the quality

\begin{tabular}{llllllll}
\hline & \multicolumn{2}{c}{$\begin{array}{c}\text { Uniformity of } \\
\text { IHC staining }\end{array}$} & \multicolumn{3}{c}{ Background staining } & \multicolumn{2}{c}{$\begin{array}{c}\text { Adequacy of } \\
\text { counter staining }\end{array}$} \\
& 0 & 1 & 0 & 1 & 2 & 0 & 1 \\
\hline ER & 42 & 83 & 24 & 89 & 7 & 14 & 111 \\
& $30.8 \%$ & $69.2 \%$ & $20.0 \%$ & $74.2 \%$ & $5.8 \%$ & $7.5 \%$ & $92.5 \%$ \\
Her2 & 36 & 87 & 17 & 93 & 8 & 13 & 110 \\
& $26.3 \%$ & $73.7 \%$ & $14.4 \%$ & $78.8 \%$ & $6.8 \%$ & $6.8 \%$ & $93.2 \%$ \\
\hline
\end{tabular}

\section{Quality of IHC staining Her-2}

IHC stained slides for Her-2 was available for only 118 cases. The best staining quality was indicated by a score of 4 as for ER. The majority, 79 (66.9\%) cases scored 3 or 4 indicating good quality of IHC staining (Table 4).

As for ER, the majority of the cases had uniform staining throughout the section and background staining did not interfere with the interpretation. Most of the cases had adequate counter staining (Table 5).

The average scores of the quality of IHC staining for ER and Her-2 were calculated separately for the 3 grades (Table 5). ANOVA was used as the statistical method to find out whether the preservation artifacts have a significant effect on the quality of IHC 
staining. Preservation artifacts did not have a statistically significant effect on the quality of IHC staining for ER $(p=0.44)$ and the quality of IHC staining for Her-2 $(p=0.50)$.

Multiple comparisons were done using Post Hoc test which did not reveal a statistically significant effect.

\section{Discussion}

Immunohistochemistry has become an integral part of histopathological diagnosis and it provides essential data predictive of clinical evolution and of therapeutic responsiveness (6). Therefore it is essential to identify factors which affect the quality of IHC staining in order to maintain the reliability of the test. There are many publications in the literature on the effects of fixation and other pre-analytical factors on the quality of staining (7). However publications on relationship between commonly encountered morphological preservation artifacts and quality of IHC staining are sparse.

For the present study we assessed the effect of commonly encountered morphological preservation artifacts (Table 1). The quality of IHC staining was assessed in terms of uniformity of staining, background staining and adequacy of counter-staining Intensity of staining was not considered as done by Maxwell and McCluggage because the intensity of ER depends on how much oestrogen receptors are expressed in the nucleus (5). Specificity of staining was also not considered as the study sample included both negative and positive breast cancers.

None of the breast cancers included in the study had score 0 for preservation artifacts. This indicates that all the specimens had at least a minor degree of morphological preservation artifacts. There was nearly one third of the total amount of cases in each Grade of preservation artifacts (Table 3). Minimum degree of preservation artifacts was present in Grade 1 and maximum degree of preservation artifacts was present in Grade 3 cases. This is inversely related to the percentage of cells that are preserved in the breast cancer sections.

Morphological preservation artifacts can be seen in the IHC stained slides because of the delay in exposure of the center of a surgical specimen to formalin (8). Most often our laboratories receive specimens, not soon after the surgery but a few hours to several days later. To avoid autolysis the whole mastectomy specimen is sent in $10 \%$ formalin. However they are unsliced and by the time they reach the pathologist the central part of the tumour may have already undergone autolysis although the gross specimen is in $10 \%$ buffered formalin due to the slow penetration of formalin. Therefore we do encounter the morphological preservation artifacts very often in the mastectomy and lumpectomy specimens although they are hard to find in Tru-cut biopsies.

Another reason for morphological preservation artifacts is dehydration which occurs in tissue processing. This is believed to be due to the difference in the consistency of tumour and stroma (9). Therefore the presence of retraction of tumour cell clusters from the stroma may have occurred, at least in some cases, during processing and not related to fixation. Cellular discohesion is a feature of poorly differentiated tumours (10). Distinction between cellular discohesion and artifactual cytoplasmic retraction can be made on the arrangement of the cell membranes of the adjacent cells. The cell membranes of the neighboring discohesive cells are randomly arranged and show cytoplasmic processes (10). We did not include the tumors with cellular separation due to discohesion into the tumors with artifacts in order to separately identify the true artifactual retraction of tumour cells.

Ibarra et al (11) have stated that delayed fixation of the center of the surgical specimen may be responsible for false-negative results because of the inadequate necessary cross-links between protein and nucleic acids required for proper IHC analysis (11). We did not assess the false negative rate in the present study.

In routine laboratory practice, pathologists examine the specimens macroscopically and microscopically and select the well preserved areas for the preparation of slides. This may be the reason why preservation artifacts were seen only in a proportion of cells in the section. Therefore the effect of preservation artifacts can be eliminated by choosing preserved areas when assessing the ER and Her-2 status.

Apart from many analytical factors antigen diffusion prior to fixation is a pre-analytical cause of background staining outside the expected antigen site (12). Most of the breast cancer cases of our study had background staining probably due to the poor 
preservation, but it did not interfere with the interpretation in the majority.

In conclusion, the commonly encountered morphological preservation artifacts; retraction of tumour cell clusters, cytoplasmic retraction and alterations in nuclear morphology do not affect the quality of IHC staining for ER and Her-2, measured in terms of uniformity of staining, background staining and adequacy of counter staining. We believe that our pilot study encourages further studies to find out the causes of morphological preservation artifacts and their effect to the quality of IHC staining.

\section{Acknowledgements}

The authors would like to thank Dr. B. Perera, Senior Lecturer, Department of Community Medicine, Faculty of Medicine, University of Ruhuna, Sri Lanka for his guidance in the statistical analysis and Mrs. G.G.D.D. Gunawardhana, Senior Staff Technical Officer, Department of Pathology, Faculty of Medicine, University of Ruhuna for the preparation of $\mathrm{H} \& \mathrm{E}$ and IHC slides.

\section{References}

1. Matos LL, Trufelli DC, de Matos MG, de Silva Pinhal MA. Immunohistochemistry as an important tool in biomarkers detection and clinical practice. Biomark Insights 2010; 5: 9-20.

2. Webster JD, Miller MA, Du Sold D, Ramos-Vara J. Effects of prolonged formalin-fixation on diagnostic immunohistochemistry in domestic animals. Journal of Histochemistry and Cytochemistry 2009; 57(8): 753-61.

3. Berod A, Hartman BK, Pujol JF. Importance of fixation in immunohistochemistry: use of formaldehyde solutions at variables $\mathrm{pH}$ for the localization of Tyrosine hydroxylase. Journal of Histochemistry and Cytochemistry 1981; 29(7): 844-50.
4. Rosai J. Auckerman's Surgical Pathology: Gross Techniques in Surgical Pathology. $8^{\text {th }}$ ed. vol 1. St Louis: Mosby, 1996: 15-6.

5. Maxwell P, McCluggage WG. Audit and internal quality control in immunohistochemistry. Journal of Clinical Pathology 2000; 53(12): 929-32.

6. Bussolati G, Leonardo E. Technical pitfalls potentially affecting diagnoses in immunohistochemistry. Journal of Clinical Pathology 2008; 61(11): 118492.

7. Mohan, H. Cell injury and cellular adaptation. Text Book of Pathology. $5^{\text {th }}$ ed, New Delhi: Jaypee Brothers Medical Publishes, 2005.

8. Khoury T, Sait S, Hwang H, Chandrasekhar R, Widing G et al. Delay to formalin fixation effect on breast biomarkers. Modern Pathology 2009; 22(11): 1457-67.

9. Spencer LT, Bancroft JD. Tissue Processing. In: Bancroft JD, Gamble M eds. Theory and Practice of Histological Techniques. 6th ed. Churchill Livingstone, 2008.

10. Roxanis I, Chow J. Cellular cohesion as a prognostic factor in malignant melanoma: a retrospective study with up to 12 years follow up. Modern Pathology 2010; 23(4): 502-10.

11. Ibarra JA, Rogers LW, Kyshtoobayeva A, Bloom K. Fixation Time Does Not Affect the Expression of Estrogen Receptor. American Journal of Clinical Pathology 2010; 133(5): 747-55.

12. Wendelboe HG, Bisgaard K. Background. In: Marc K, ed. Immunohistochemical Staining Methods. $4^{\text {th }}$ ed. DAKO Cytomation, Carpinteria: California, 2006. 